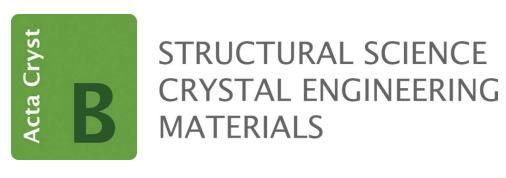

ISSN 2052-5206
Keywords: quantum crystallography; electron density; X-ray diffraction; wavefunctions.
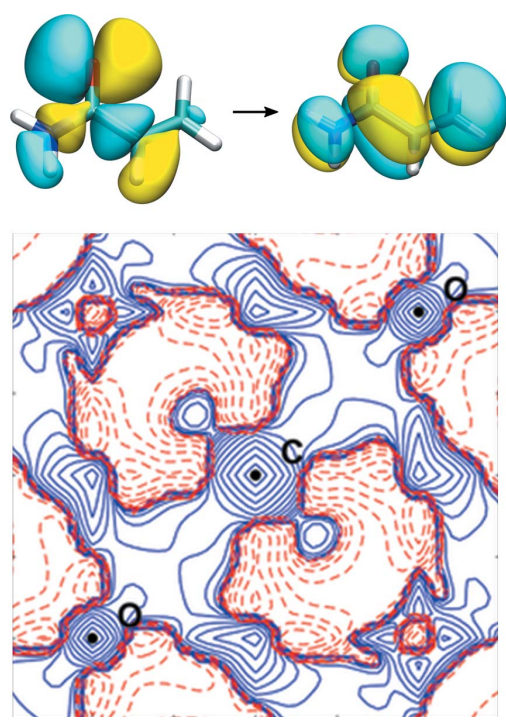

\section{Quo vadis, quantum crystallography?}

\author{
Jean Michel Gillet ${ }^{\mathrm{a} *}$ and Piero Macchi ${ }^{\mathrm{b} *}$
}

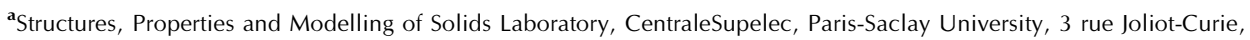
Gif-sur-Yvette, 91191, France, and ${ }^{\mathbf{b}}$ Chemistry, Materials and Chemical Engineering, Politecnico di Milano, via Mancinelli 7, Milano, 20131, Italy. *Correspondence e-mail: jean-michel.gillet@centralesupelec.fr, piero.macchi@polimi.it

In the past few issues of Acta Crystallographica Section B, including the current one, several articles have reported research work on quantum crystallography. Collectively they comprise a virtual special issue on the subject, the first on such an extended concept in the IUCr journals. Previously, a special issue, dedicated to Philip Coppens (1930-2017), was published in Acta Crystallographica Section B (August 2017). It contained many contributions in the field of charge density (as well as photo-crystallography). Originally intended to celebrate his retirement with contributions on two of the main topics developed during his career, the issue appeared just a few weeks after Professor Coppens passed away and became a kind of memorial issue.

The occasion of the present special issue is the principal authors' participation in the first online quantum crystallography meeting (QCrOM2020) held in August 2020. It was organized (and mostly improvised) to replace the sessions on this subject initially programmed for the IUCr Congress in Prague, which, as we know, was postponed to 2021. Its virtual modality (and its subsequent free of charge registration) made it possible to attract attendees from a wider range of expertise. It was the opportunity to present the latest results and reviews on the field and share opinions during fruitful discussion sessions that normally do not take place at large scale and tightly scheduled meetings like those of the IUCr Congress.

Despite all the difficulties caused by the pandemic the field is currently blooming, and the community is undergoing a generational turnover with many new young researchers involved and new groups established. The field's momentum is testified by the rather broad spectrum of studies published in this special issue, with a variety of research themes and many topics analyzed or reviewed in detail.

Quantum crystallography is a modern name for a field that started when quantum mechanics itself was put forward, coinciding with the early days of X-ray crystallography. In keeping with Peter Debye's early intuition (Debye, 1915), the discovery of X-ray diffraction offered a whole new possibility 'to establish by experiment the particular arrangement of the electrons in the atoms'. Many studies became possible thanks to the interplay between crystallographic techniques and quantum physics. For example, experimental crystallography was used to unveil the nature of electrons (waves and corpuscles; see De Broglie, 1929), to investigate the electronic structures of metals (Weiss \& Demarco, 1958), to map the charge density around atoms to form bonds and molecules or solids (Coppens, 1967), and the electron polarization upon application of external stimuli (such as the electric field, see Hansen et al., 2004) or upon temperature changes.

Quite remarkably, these kinds of studies are those that originally attracted the interest of quantum physicists for the emerging field of X-ray crystallography in the 1920s. At the same time, chemists also envisaged the exceptional outcome from crystallographic studies and the vast array of details useful in developing theories of chemical bonding (Pauling, 1939).

This sentiment has always accompanied studies on accurate charge density, which has become the favourite observable for revealing the nature of chemical bonding and the supramolecular interactions, especially - but not only - within the paradigm of the quantum theory of atoms in molecules (Bader, 1990) that becomes, in the crystallographic framework, the quantum theory of atoms in molecules and crystals (Gatti, 2005). 
In the past two decades, and especially in recent years, we have witnessed a renewal of the interest in a direct determination of the crystal wavefunction, which has indeed been a goal since the beginning of the X-ray diffraction era (Pauling, 1926). For example, there is growing attention being given to calculations of wavefunctions restrained to reproduce the X-ray diffracted intensities (Jayatilaka, 1998), as well as to the refinement of orbital coefficients (Tanaka et al., 2008) or of reduced-density matrices (Gillet, 2007).

A search in the database of papers published in Acta Crystallographica Section B since 1998 (online versions), returns more than 500 papers directly or indirectly related to quantum crystallography (and overall, $\sim 4000$ in all IUCr journals). Most of them report accurate charge density studies and their applications, encompassing the technological progress which has occurred over the years, such as the availability of area detectors, the development of synchrotron sources and low-temperature devices (see for example, Iversen et al., 1999). Moreover, a discussion on the future of topological analysis of experimental charge density took place (Dittrich, 2017; Macchi, 2017).

The virtual special issue that we have edited seamlessly combines the recent developments and an outlook on future research in this field, highlighting fundamental questions, scientific cases and broad applications in other disciplines.

Despite the growing appeal of molecular/crystal orbital calculations, orbital-free quantum crystallography is emerging, in keeping with the emergence of orbital-free density functional theory (see Finzel, 2021; Tsirelson \& Stash, 2021; Bartashevich et al., 2021).

While quantum crystallography often deals with molecular aspects of electron distribution, the role of environment and of intermolecular interactions is being ever more scrutinized (see Zheng et al., 2021; Macetti et al., 2021; Bergmann et al., 2021; Forni et al., 2021; Kleemiss et al., 2021).

Although scientists often think of electron density as the mere distribution of charge in a molecule/crystal, one should not forget that electrons do have a spin as well and that molecular or extended systems may feature an overall spin magnetic moment. Thus spin-density models are essential (see Souhassou et al., 2021), especially in view of the increasing development of spintronics. The paper by Souhassou et al. (2021) also deals with the refinement of orbital coefficients in inorganic solids, another frontier topic. The role of ligand coordination for setting the oxidation state of a metal is also discussed by Adamko Koziskova et al. (2021).

Landeros-Rivera, Contreras-García \& Dominiak (2021) discuss refinement strategies in electron wavefunctions obtained from X-ray diffraction data while Launay \& Gillet (2021) deal with the $N$-representability of reduced density matrices reconstructed from several X-ray scattering techniques. Shteingolts et al. (2021) instead discuss transferable aspherical atom models for improving the quality of refinements.

One of the appeals of quantum crystallographic techniques, especially in the past few years, is the possibility of using very accurate modelling (such as atomic multipolar coefficients and molecular wavefunctions) to improve the standard (bio)chemical crystallographic studies. Such a link is fundamental because quantum crystallography should be intimately connected with the rest of crystallography and all related sciences. The quest for highly accurate observations and ever more precise calculations, inherent to the most fundamental aspects of quantum crystallography, has many implications for applied science and technology. In this respect, the present state bears little difference to quantum mechanics, originally developed as a very sophisticated theory for elite scientists, which has proved to be vital for several technological applications, up to quantum computing.

We are confident that this special issue will contribute to attracting ever-growing interest not only in the fundamental bases of quantum crystallography, but also in its applicative aspects. We also thank all the authors of contributions to this special issue for their dedication and excellent work, and the Main Editors of Acta Crystallographica Section B for this opportunity.

The virtual special issue is available at https://journals.iucr.org/special_issues/2021/QCrOM2020.

\section{Acknowledgements}

We would like to acknowledge the excellent work accomplished by Sara Delahaie, Hugo Julien, Antoine Marras and Nizar Melk, who were - at the time - first year engineering students at CentraleSupelec. Their enthusiasm and hard work were decisive in the organization (in such a short timeframe) of the first online quantum crystallography meeting (QCrOM2020). We also thank CentraleSupelec for their permission to use their logo for the conference (and the cover page of this issue) as well as for their essential financial and technical support.

\section{References}

Adamko Koziskova, J. A., Chen, Y.-S., Grass, S.-Y., Chuang, Y.-C., Hsu, I-J., Wang, Y., Lutz, M., Volkov, A., Herich, P., Vènosovà, B., Jelemenskà, I., Bucinsky, L., Breza, M. \& Kozisek, J. (2021). Acta Cryst. B77, 919-929.

Bader, R. F. W. (1990). Atoms in Molecules: A Quantum Theory. International Series of Monographs on Chemistry 22. Oxford: Oxford Science Publications.

Bartashevich, E., Stash, A., Yushina, I., Minyaev, M., Bol'shakov, O., Rakitin, O. \& Tsirelson, V. (2021). Acta Cryst. B77, 478-487.

Bergmann, J., Oksanen, E \& Ryde, U. (2021). Acta Cryst. B77, 906918.

Coppens, P. (1967). Science, 158, 1577-1579.

De Broglie, L. (1929). The Wave Nature of the Electron. Nobel Lecture.

Debye, P. (1915). Ann. Phys. 351, 809-823.

Dittrich, B. (2017). Acta Cryst. B73, 325-329.

Finzel, K. (2021). Acta Cryst. B77, 458-466.

Forni, A, Cariati, E., Carlucci, L., Lucenti, E., Marinotto, D., Pieraccini, S. \& Sironi, M. (2021). Acta Cryst. B77, 865-870.

Gatti, C. (2005). Z. Kristallogr. 220, 399-457.

Gillet, J. M. (2007). Acta Cryst. A63, 234-238.

Hansen, N. K., Fertey, P. \& Guillot, R. S. (2004). Acta Cryst. A60, 465471.

Iversen, B. B., Larsen, F. K., Pinkerton, A. A., Martin, A., Darovsky, A. \& Reynolds, P. A. (1999). Acta Cryst. B55, 363-374. 
Jayatilaka, D. (1998). Phys. Rev. Lett. 80, 798-801.

Kleemiss, F., Puylaert, P., Duvinage, D., Fugel, M., Sugimoto, K., Beckmann, J. \& Grabowsky, S. (2021). Acta Cryst. B77, 892-905.

Landeros-Rivera, B., Contreras-García, J. \& Dominiak, P. M. (2021). Acta Cryst. B77, 715-727.

Launay, Y. \& Gillet, J.-M. (2021), Acta Cryst. B77, 683-694.

Macchi, P. (2017). Acta Cryst. B73, 330-336.

Macetti, G., Macchi, P. \& Genoni, A. (2021). Acta Cryst. B77, 695705.

Pauling, L. (1926). Letter to A. A. Noyes. Linus Pauling and The Nature of the Chemical Bond: A Documentary History website. https://scarc.library.oregonstate.edu/coll/pauling/bond/corr/ corr278.1-lp-noyes-19260425.html
Pauling, L. (1939). The Nature of the Chemical Bond, 1st ed. New York: Cornell University Press.

Shteingolts, S. A., Voronina, J. K., Saifina, L. F., Shulaeva, M. M., Semenov, V. E. \& Fayzullin, R. R. (2021). Acta Cryst. B77, 871-891.

Souhassou, M., Kibalin, I., Deutsch, M., Voufack, A. B., Lecomte, C. \& Claiser, N. (2021). Acta Cryst. B77, 706-714.

Tanaka, K., Makita, R., Funahashi, S., Komori, T. \& Win, Z. (2008). Acta Cryst, B64, 437-449.

Tsirelson, V. \& Stash, A. (2021). Acta Cryst. B77, 467-477.

Weiss, R. J. \& Demarco, J. J. (1958). Rev. Mod. Phys. 30, 59-62.

Zheng, K., Li, D., Jiang, L., Li, X., Xie, C., Feng, L., Qin, J., Qian S. \& Pang Q. (2021). Acta Cryst. B77, 311-320. 